\title{
Correlation between the overexpression of urokinase receptor isoform UPAR (D1D2) and hepatic cell malignant transformation
}

\author{
YINGQUN ZHOU ${ }^{1}$, XIAOPING LÜ${ }^{2}$, SHAN LI $^{1}$ and LINGLING ZHAN ${ }^{1}$ \\ Departments of ${ }^{1}$ Clinical Laboratory and ${ }^{2}$ Gastroenterology, The First Affiliated Hospital of Guangxi Medical University, \\ Nanning, Guangxi 530021, P.R. China
}

Received August 6, 2013; Accepted February 2, 2014

DOI: $10.3892 / \mathrm{mmr} .2014 .2006$

\begin{abstract}
In order to provide an abundant source of specimen and to reveal the correlation between the overexpression of urokinase plasminogen activator receptor isoform uPAR (D1D2) and hepatic cell malignant transformation, the optimal liver cell culture method was selected from three cell culture methods to culture and separate out liver cells with a high density, high purity and high activity. The specimens were used to culture and assess the uPAR (D1D2) mRNA level in normal liver cells, liver cancer cells and para-carcinoma cells. In the present study, the correlation between the overexpression of uPAR (D1D2) and hepatic cell malignant transformation was discussed. When comparing the tissue block adherent method, liver cell grinding method and pancreatic enzyme digestion method, the liver tissue adherent method was found to be economical, simple and overall the optimal method for liver cell culture. This was used as a reference standard for cell culture. RT-PCR was used to determine isoform uPAR (D1D2) mRNA level in normal liver cells, para-carcinoma cells and liver cancer cells. The comparison of uPAR (D1D2) mRNA levels in normal liver cells, para-carcinoma cells and liver cancer cells, demonstrated that the brightness of the cells clearly increased in normal liver cells, para-carcinoma cells and liver cancer cells. The comparison of the cell grey values of three groups demonstrated a statistically significant difference $(\mathrm{P}<0.05)$. The liver tissue adherent method was able to produce liver cells with a high density, high purity and high activity, providing a sufficient source of specimen for our
\end{abstract}

Correspondence to: Professor Lingling Zhan, Department of Clinical Laboratory, The First Affiliated Hospital of Guangxi Medical University, 22 Shuangyong Road, Nanning, Guangxi 530021, P.R. China

E-mail: 13197614098@163.com

Key words: liver cell culture, urokinase plasminogen activator receptor isoform, cell signal conduction abnormalities, liver cell malignant transformation subsequent experiments. The electrophoresis results showed that: uPAR (D1D2) mRNA expression increased from normal liver cells to para-carcinoma cells to liver cancer cells, inferring that UPAR (D1D2) mRNA overexpression may be the result of changes in the conformation of the UPAR isoform. In addition, it is closely associated with abnormal cell signal transduction, which leads to clonal proliferation and abnormal differentiation of liver cells with malignant transformation in liver cells.

\section{Introduction}

Hepatocellular carcinoma (HCC) has one of the highest incidence rates of malignant tumors, ranking fifth in the world, with one-third occurring in China, where each year more than a half of newly suffering HCC patients are Chinese.

The occurrence of HCC is associated with numerous factors. Tumor incidence, development and metastasis are not only the result of the effect of certain tumor cells, but also the result of multiple systems and multiple factor effects due to the internal tumor environment. Several studies have demonstrated that the urokinase plasminogen activator receptor (UPAR) is a multifunctional receptor. It has been verified that UPAR affects cell proliferation, migration and adhesion, and its expression is associated with the malignant degree of a tumor (1). The interaction of UPAR with integrin $\alpha 5 \beta 1$, a fibronectin receptor, is able to activate FAK and ERK. In the process of microfiber formation, uPAR induces integrin $\alpha 5 \beta 1$ to combine with fibronectin and form insoluble aggregates. The uPAR signal conduction pathway mediated by integrin $\alpha 5 \beta 1$ can promote tumor cell proliferation and participate in the regulation of cell proliferation and differentiation (2). Current evidence also suggests that the MAPK signaling pathway is a major way of activating the receptor. uPAR is a highly glycosylated single-chain glycoprotein, which has three homologous domains, D1, D2 and D3. uPA can dissociate three types of isoform, including uPAR (D1), uPAR (D2D3), uPAR (D1D2)and uPAR (D3) (3). It has been verified that D1D2 is able to bind ligands, however, its ligand affinity is lower than full suPAR. D3 has an important role in managing the receptor with high affinity (4). However, the analysis of biomolecular interactions demonstrates that the 
lack of affinity is due to the increase in the dissociation rate of the D1D2 ligand complex (5). uPAR may affect multiple biological functions, including migration, adhesion, tumorigenicity and differentiation. The combination of UPAR with extracellular proteins and its interaction with certain membrane receptors, including the integrin and epidermal growth factor receptor, is a new focus of study (6).

At present, the number of different types of splice variants of UPAR that exist and their underlying mechanisms in the development of tumorigenesis remains to be elucidated. Therefore, in the present study, following the comparison of the organization block adherent method, the liver cell grinding method and the pancreatic enzyme digestion method, one in vitro cultivation method which was the most suitable and the most similar to the body cell growth environment was selected. Then, the uPAR splice variants exon D1D2 was determined by RT-PCR and its expression level in liver cancer development was discussed. The present study aimed to determine the UPAR splice variants exon D1D2 and examine the role of UPAR exons D1D2 in the process of liver cancer. This may provide the theoretical basis for clinical diagnosis, prognosis and treatment.

\section{Materials and methods}

Experimental materials. The study was approved by the Medical Ethics Committee of the First Affiliated Hospital of Guangxi Medical University (Nanning, China). Written informed consent was obtained from the patient or the families of the patients. Following signing of the informed ethical consent forms, the surgically resected normal liver tissue, tissue adjacent to liver cancer and liver cancer tissue were collected from five cases of patients with hepatic hemangioma and 30 cases of HCC at the Department of Hepatobiliary Surgery of The First Affiliated Hospital of Guangxi Medical University. The separated tissues were immediately preserved in DMEM medium at $4^{\circ} \mathrm{C}$ and the following experiments were completed rapidly.

The selected liver cell culture method was used to collect normal liver cells, para-carcinoma cells and liver cancer cells of a high density, high purity, high activity and to examine the uPAR (D1D2) level.

Instruments and reagents. DMEM medium, calf serum (Hyclone, Logan, UT, USA), trypan blue (Sigma, St. Louis, MO, USA), mycoplasma fetal bovine serum, $0.25 \%$ pancreatic enzyme (Hangzhou Sijiqing Biological Engineering Materials Co., Ltd., Hangzhou, Zhejiang, China), a $\mathrm{CO}_{2}$ incubator (Thermo Fisher Scientific, Rockford, IL, USA), an inverted microscope (Nikon, Tokyo, Japan), $4^{\circ} \mathrm{C}$ refrigerator (Haier Group, Qingdao, Shandong, China) and a tissue culture plate (Corning Inc., Corning, NY, USA) were used in the present study.

Main instruments and reagents. An ultraviolet spectrophotometer (NanoDrop 2000; Nanodrop Corporation, Wilmington, DE, USA), ultra-low temperature freezer (Thermo Fisher Scientific), Gel Doc XP + Gel imaging system (Bio-Rad, Hercules, CA, USA), voltage steady flow electrophoresis apparatus (DYY-8B; Beijing Liuyi Instrument Plant, Beijing,
China), thermal cycling machine (ABI Veriti; Applied Biosystems, Foster, CA, USA), TRIzol, RNA PCR kit and nucleic acid dye (Qianjiang Green Sea Treasure Biological Technology Co., Ltd., Qianjing, Hubei, China), PCR kit and agarose (Thermo Fisher Scientific) were used in the present study. The primer was designed by Shanghai Biotechnology Co. (Shanghai, China) and synthesized by Invitrogen Trading Co. Ltd., (Shanghai, China; Table I)

Liver cell culture method. The pancreatic enzyme digestion method was as follows. Fresh liver tissue was collected from hepatic hemangioma patients by surgical resection and removing the capsule and the blood vessels. The liver tissue was cut into $\sim 1.0 \mathrm{~cm}^{3}$ pieces in aseptic conditions and washed two to three times with PBS solution. The tissue was then cut using ophthalmic scissors and washed again with PBS solution until it turned white. Next, it was centrifuged $(560 \mathrm{x} \mathrm{g}$; $5 \mathrm{~min}$ ) and the supernatant was decanted and discarded. Trypsin (0.25\%), which was 10-15 times tissue, was mixed with tissue in a tube and then placed in $37^{\circ} \mathrm{C}$ water for 8-10 $\mathrm{min}$. The tube was agitated every few minutes to ensure the tissue was in full contact with the digestive enzymes. Following agitation, the supernatant was discarded and $10 \mathrm{ml}$ of DMEM medium containing $10 \%$ calf serum was added into the tube to terminate digestion and the solution was gently pipetted to disperse the tissue. Following centrifugation (560 x g; $5 \mathrm{~min}$ ), the supernatant was discarded using a 200-hole stainless steel mesh filter. DMEM (1.0 ml) was added into the tube again and gently blown in order to make the cell suspension. Trypan blue was added and the cells were counted. The proportion of live cells was $\sim 90 \%$. The cell suspension was placed equally into three tubes $(0.1 \mathrm{ml} /$ tube $)$ and the appropriate amount of DMEM containing $(10,15$ or $20 \%$ ) calf serum (mycillin: medium $=1: 100$ ) was added. It was then blended well, setting the cell concentration to $1 \times 10^{6} / \mathrm{ml}$. The cell suspension was plated at a density of $2 \times 10^{5} / \mathrm{cm}^{2}$ and then the cell culture plate was placed in an incubator of $5 \%$ $\mathrm{CO}_{2}$ at $37^{\circ} \mathrm{C}$.

Liver cell grinding method. Fresh liver tissue was collected from the hepatic hemangioma patients by surgical resection and removing the capsule and the blood vessels. The liver tissue was cut into $\sim 1.0 \mathrm{~cm}^{3}$ pieces in aseptic conditions and was washed two to three times with PBS solution. The tissue was then cut using ophthalmic scissors and washed again with PBS solution until the color turned white. Then the tissue which had been cut up was transferred into a 200-hole stainless steel mesh filter and the tissue was gently ground repeatedly using a $10 \mathrm{ml}$ glass syringe. DMEM medium was added constantly in order to prevent the loss of tissue moisture in the process. The filtrated cell suspension was centrifuged (560 x g; $5 \mathrm{~min}$ ) and the supernatant was discarded. DMEM $(1.0 \mathrm{ml})$ was added and the cell suspension was gently blown. The trypan blue was added and the cells were counted. The proportion of live cells was $\sim 90 \%$. The cell suspension was equally placed into three tubes $(0.1 \mathrm{ml} /$ tube $)$ and the appropriate amount of DMEM containing (10, 15 or 20\%) calf serum (mycillin: medium=1:100) was added. It was then blended well, setting the cell concentration to $1 \times 10^{6} / \mathrm{ml}$. The cell suspension was plated at a density of $2 \times 10^{5} / \mathrm{cm}^{2}$ and then 
Table I. RT-PCR primers.

\begin{tabular}{llll}
\hline Gene & Length & Primer & Sequence \\
\hline uPAR (D1D2) & $367 \mathrm{bp}$ & Forward & 5'-GACCTCTGCAGGACCACGAT-3' \\
GAPDH & & Reverse & 5'-GGTGGCGGTCATCCTTTG-3' \\
& $292 \mathrm{bp}$ & Forward & 5'-GGTGCTGAGTATGTCGTGGAG-3' \\
& & Reverse & 5'-CAGTCTTCTGAGTGGCAGTGAT-3'
\end{tabular}

uPAR, urokinase plasminogen activator receptor isoform; RT-PCR, reverse transcription polymerase chain reaction.

the cell culture plate was placed in an incubator of $5 \% \mathrm{CO}_{2}$ at $37^{\circ} \mathrm{C}$.

Tissue block adherent method. Fresh liver tissue was collected from hepatic hemangioma patients by surgical resection and removing the capsule and the blood vessels. The liver tissue was cut into $\sim 1.0 \mathrm{~cm}^{3}$ pieces in aseptic conditions and washed two to three times with PBS solution. The tissue was then cut using ophthalmic scissors and washed again with PBS solution until the color turned white. Following centrifugation (560 x g; $5 \mathrm{~min}$ ) the supernatant was decanted and discarded. Trypsin $(0.25 \%)$ was added to the test tube and mixed, and then the tube was placed in $37^{\circ} \mathrm{C}$ water for $8-10 \mathrm{~min}$. The tube was agitated every few minutes to ensure that the tissue was in full contact with the digestive enzymes. Following agitation, the supernatant was discarded and $10 \mathrm{ml}$ of DMEM medium containing $10 \%$ calf serum was added into the tube to terminate digestion, and the solution was gently pipetted to disperse the tissue. Following centrifugation (560 x g; 5 min), the supernatant was discarded using a 200-hole stainless steel mesh filter. DMEM $(1.0 \mathrm{ml})$ was added into the tube again and gently blown in order to make the cell suspension. Trypan blue was added and the cells were counted. The proportion of live cells was $\sim 90 \%$. The cell suspension was equally placed into three tubes $(0.1 \mathrm{ml} /$ tube $)$ and the appropriate amount of DMEM containing (10, 15 or $20 \%$ ) calf serum (mycillin: medium $=1: 100$ ) was added and blended well. Tissue blocks were distributed evenly in the cell culture plate and it was ensured that the tissue would not float on the cell culture plate. The tissues were then cultured in an incubator of $5 \% \mathrm{CO}_{2}$ at $37^{\circ} \mathrm{C}$. It was forbidden to agitate the cell culture plate for $72 \mathrm{~h}$ from the beginning of the cell culture otherwise the tissue block was unable to adhere to the wall of the cell culture plate, which affects cell proliferation.

Observation of cell morphology, and testing of the cell survival rate, purity and the albumin level of the culture supernatant. Cell morphological changes were observed using an inverted microscope in the culture process. Liver cells were dyed with the routine method of PAS staining as the liver cells contain plenty of glycogen granules which can be dyed red and the nucleus is vacuolated. The cell purity and survival rate of the three types of cells with the three methods were calculated under the inverted microscope. Culture supernatants were collected and the albumin level was tested prior to replacement of the cell culture fluid each time.
Comparing the three methods of cultivation and selecting the most suitable normal liver tissue culture method as a reference standard. Specimen carcinoma tissues and adjacent tissues of 30 cases of the HCC patients who survived liver cancer surgery were collected. The diagnostic criteria of liver cancer was set according to the criteria which was revised at the 8th National Academic Conference of Liver Cancer in September 2001. Among the thirty HCC patients, 28 were male and two were female, aged between 25-65 years old with an average age of 49 years old. The cell culture method referred to the one which was the most suitable for normal liver cell culture.

RNA extraction. TRIzol ( $1 \mathrm{ml})$ was added into the cell culture bottle and the bottle was placed on ice for 5-10 min. The cell lysates in the bottle were suctioned into the $1.5 \mathrm{ml} \mathrm{EP}$ tubes and $0.2 \mathrm{ml}$ of chloroform was added to every tube. The tubes were turned upside down, blended and kept at room temperature for $5 \mathrm{~min}$. Then, the tubes were centrifuged for $15 \mathrm{~min}(80,486 \mathrm{x} \mathrm{g}$ at $4^{\circ} \mathrm{C}$. The sublayer contained DNA, the white middle layer contained protein and the upper layer contained RNA. The RNA in the upper layer, which was colorless liquid, was moved into another EP tube and isopycnic dimethyl carbinol was added. The tube was blended, kept at room temperature for $10 \mathrm{~min}$ and then centrifuged for $15 \mathrm{~min}(80,486 \mathrm{x} \mathrm{g})$ at $4^{\circ} \mathrm{C}$. For the precipitation of RNA, the supernatant was poured out and the white sediment was kept. Then, it was added to $1 \mathrm{ml}$ of $75 \%$ absolute ethyl alcohol, beaten gently, centrifuged at $55,890 \times \mathrm{g}$ at $4^{\circ} \mathrm{C}$ for $10 \mathrm{~min}$, and then the alcohol was carefully discarded. The tube of RNA was centrifuged at $55,890 \mathrm{x}$ g at $4^{\circ} \mathrm{C}$ for $10 \mathrm{~min}$ again, the alcohol was carefully discarded, dried in the air for $10 \mathrm{~min}$ and added to the appropriate amount of DEPC (D100T) to dissolve the RNA. An ultraviolet spectrophotometer was used to measure the RNA concentration and the OD numerical value. The RNA purity was good if the OD260/280 numerical value was between 1.8 and 2.0. Reverse transcription was performed according to the manufacturer's instructions.

Statistical analysis. The cell survival rate, cell purity and the ratio of albumin to grey scale of cell culture supernatant are expressed as the means \pm standard deviation (SD) and were compared between the two groups. Student's t-test was used to compare the difference between the patients' characteristics. SPSS software version 17.0 (SPSS, Inc., Chicago, IL, USA) was used. $\mathrm{P}<0.05$ was considered to indicate a statistically significant difference. 
Table II. Comparision of cell purity, survival rates and supernatant albumin levels of normal liver tissue in three cultivation methods with differing serum concentrations.

\begin{tabular}{|c|c|c|c|c|}
\hline \multirow[b]{2}{*}{ Culture methods } & \multirow{2}{*}{$\begin{array}{l}\text { Cell parameters of } \\
\text { different culture methods }\end{array}$} & \multicolumn{3}{|c|}{ Serum concentrations } \\
\hline & & $10 \%$ & $15 \%$ & $20 \%$ \\
\hline \multirow[t]{3}{*}{ Pancreatic enzyme digestion } & Survival rates $(\%)$ & $47.75 \pm 0.67$ & $56.80 \pm 1.63^{\mathrm{a}, \mathrm{b}}$ & $46.42 \pm 1.40$ \\
\hline & Cell purity $(\%)$ & $58.08 \pm 0.94$ & $58.96 \pm 0.75$ & $58.48 \pm 0.61$ \\
\hline & Supernatant albumin levels (g/l) & $0.95 \pm 0.07$ & $3.1 \pm 0.13^{\mathrm{a}, \mathrm{b}}$ & $2.4 \pm 0.08$ \\
\hline \multirow[t]{3}{*}{ Liver cell grinding } & Survival rates $(\%)$ & $64.68 \pm 1.05$ & $71.82 \pm 0.77^{\mathrm{a}, \mathrm{b}}$ & $66.62 \pm 0.72$ \\
\hline & Cell purity $(\%)$ & $79.20 \pm 0.35$ & $80.20 \pm 0.60$ & $80.16 \pm 1.27$ \\
\hline & Supernatant albumin levels (g/l) & $1.35 \pm 0.05$ & $3.86 \pm 0.08^{\mathrm{a}, \mathrm{b}}$ & $2.8 \pm 0.08$ \\
\hline \multirow[t]{3}{*}{ Tissue block adherent } & Survival rates $(\%)$ & $79.66 \pm 0.74$ & $90.16 \pm 0.88^{\mathrm{a}, \mathrm{b}}$ & $83.08 \pm 0.65$ \\
\hline & Cell purity $(\%)$ & $86.34 \pm 0.78$ & $86.68 \pm 0.73$ & $87.50 \pm 0.46$ \\
\hline & Supernatant albumin levels (g/l) & $2.20 \pm 0.06$ & $4.21 \pm 0.04^{\mathrm{a}, \mathrm{b}}$ & $3.86 \pm 0.08$ \\
\hline
\end{tabular}

${ }^{\mathrm{a}} \mathrm{P}<0.05$, comparison between 15 and $10 \%$ serum concentrations; ${ }^{\mathrm{b}} \mathrm{P}<0.05$, comparison between 15 and $20 \%$ serum concentrations.

Table III. Comparison of the cell survival rate, purity and albumin supernatant fluid in three different cultivation methods.

\begin{tabular}{lccc}
\hline Methods & Survival rates (\%) & $\begin{array}{c}\text { Supernatant } \\
\text { Cell purity (\%) }\end{array}$ & $\begin{array}{c}\text { albumin levels (g/l) } \\
\text { Pancreatic enzyme digestion }\end{array}$ \\
Liver cell grinding & $56.80 \pm 1.63$ & $58.96 \pm 0.75$ & $3.1 \pm 0.13$ \\
Tissue block adherent & $71.82 \pm 0.77$ & $80.20 \pm 0.60$ & $3.86 \pm 0.08$ \\
& $90.16 \pm 0.88^{\mathrm{a}, \mathrm{b}}$ & $86.68 \pm 0.73$ & $4.21 \pm 0.04^{\mathrm{a}, \mathrm{b}}$
\end{tabular}

${ }^{\mathrm{a}} \mathrm{P}<0.05$, comparison with pancreatic enzyme digestion method; ${ }^{\mathrm{b}} \mathrm{P}<0.05$, comparison with liver cell grinding method.

\section{Results}

Liver cell morphology. The liver cell morphology was observed using an inverted microscope when the liver cells were colored by the conventional PAS staining method (Fig. 1). The cell purity and survival rate was observed using an inverted microscope and the albumin level of cell supernatant is listed in Table II.

Pancreatic enzyme digestion method. There were a small amount of cells, which adhered to the wall of the tube the next day and the majority of the adherent cells were epithelial cells. The growth conditions of the cells were better in the medium with a serum concentration of $15 \%$ than different serum concentrations. However, there were small amounts of liver cells and a large number of fibroblasts and mesenchymal cells in the culture plate, and the number of cells failed to meet the requirement of the experiment (Fig. 2A1-A3).

Liver cell grinding method. Following cell culture, numerous cell fractions were identified in the culture plate and there were more adherent cells. There was a small amount of cells which had adhered to the wall the next day, however, the majority of them were epithelial cells. The growth conditions of the cells were better in the medium with a serum concentration of $15 \%$ compared with the other serum concentrations. However, the cells grew slowly and the total number of cells failed to meet the requirements of the experiment (Fig. 2B1-B3).

Tissue block adherent method. Following 3 days, there was outgrowth which surrounded the tissue block. Cell growth gradually spread from the tissue block to the surrounding area. Under the light microscope, the liver cell shape appeared trianglular, round or quasicircular. The cells were arranged in neat rows and their boundaries were clear and rich in cytoplasm. The nucleus was clearly visible. Certain cells were mononuclear and the others were dinuclear, in a circular or elliptical shape. Following generation, a large quantity of liver cells with high purity and good activity were produced (Fig. 2C1-C3).

By comparing the results, it was found that the cell survival rate and cell purity were the highest when the tissue block adherent method was used, and the most suitable serum concentration was $15 \%$ (Table II).

The normal liver tissues from 5 cases of hemangiomas were separately cultured by use of the three serum concentrations and the three types of cell culture methods. The albumin level in the liquid supernatant was determined prior to changing the liquid supernatant. The results of the albumin levels are listed in Table II.

The results of a comparison of cell purity, survival rates and supernatant albumin levels in normal liver tissues of 5 cases of patients with hepatic hemangioma with three 
Table IV. Expression of uPAR (D1D2) in three groups.

\begin{tabular}{lcc}
\hline Group & $\mathrm{n}$ & Grey value ratio \\
\hline Cancer cell & 28 & $0.845 \pm 0.291^{\mathrm{a}}$ \\
Para-carcinoma cell & 28 & $0.525 \pm 0.244^{\mathrm{a}}$ \\
Control group & 10 & $0.293 \pm 0.035^{\mathrm{a}}$
\end{tabular}

Grey level ratio [uPAR (D1-D2)/GAPDH] was used as the gene expression level. ${ }^{\mathrm{a}} \mathrm{P}=0.000$.

Table V. Relationship between the expression of uPAR (D1D2) in $\mathrm{HCC}$ and clinical pathological features.

\begin{tabular}{lcc}
\hline Pathological features & $\mathrm{n}$ & $\begin{array}{c}\text { uPAR (D1D2) } \\
\text { grey value ratio }\end{array}$ \\
\hline $\begin{array}{l}\text { AFP }(\mu \mathrm{g} / \mathrm{ml}) \\
>400\end{array}$ & 13 & $0.935 \pm 0.305$ \\
$<400$ & 15 & $0.773 \pm 0.265$ \\
SF $(\mu \mathrm{g} / \mathrm{l})$ & & \\
$>400$ & 11 & $0.859 \pm 0.385$ \\
$<400$ & 17 & $0.842 \pm 0.223$ \\
DNA copy number & & \\
$<1.0 \times 10^{3}$ & 8 & $0.692 \pm 0.105$ \\
$>1.0 \times 10^{3}$ & 20 & $0.911 \pm 0.318^{\mathrm{a}}$ \\
Pathological grading & & \\
1 & 6 & $0.705 \pm 0.169$ \\
$2+3$ & 22 & $0.887 \pm 0.307$ \\
Tumor size (cm) & & $0.843 \pm 0.223$ \\
$<5$ & 14 & $0.854 \pm 0.354$ \\
$>5$ & 14 & \\
\hline
\end{tabular}

${ }^{\mathrm{a}} \mathrm{P}=0.012$. AFP, $\alpha$-fetoprotein.

different cultivation methods at a serum concentration of $15 \%$ are shown in Table III.

By comparison, it was revealed that the tissue adherent method with a serum concentration of $15 \%$ was the optimal method for liver cell culture. With reference to the optimal culture method of normal liver tissue (tissue block adherent method), the present study further determined urokinase receptor isoform exons D1D2 in liver cancer cells and adjacent cells in 30 cases of HCC by cell culture.

The concentration and purity of RNA extracted from cells was determined by an ultraviolet spectrophotometer. The A260/280 results were between 1.8 and 2.0, which suggested that the RNA had a high purity. Thus, the RNA conformed to the requirements of the reverse transcription and $-80^{\circ} \mathrm{C}$ preservation.

The expression of UPAR (D1D2) mRNA of liver cancer cells, para-carcinoma cells and normal cells are shown in Fig. 3. From electrophoresis, it was apparent that UPAR (D1D2) was expressed in cancer cells, para-carcinoma cells and normal cells due to the appearance of a well-distributed
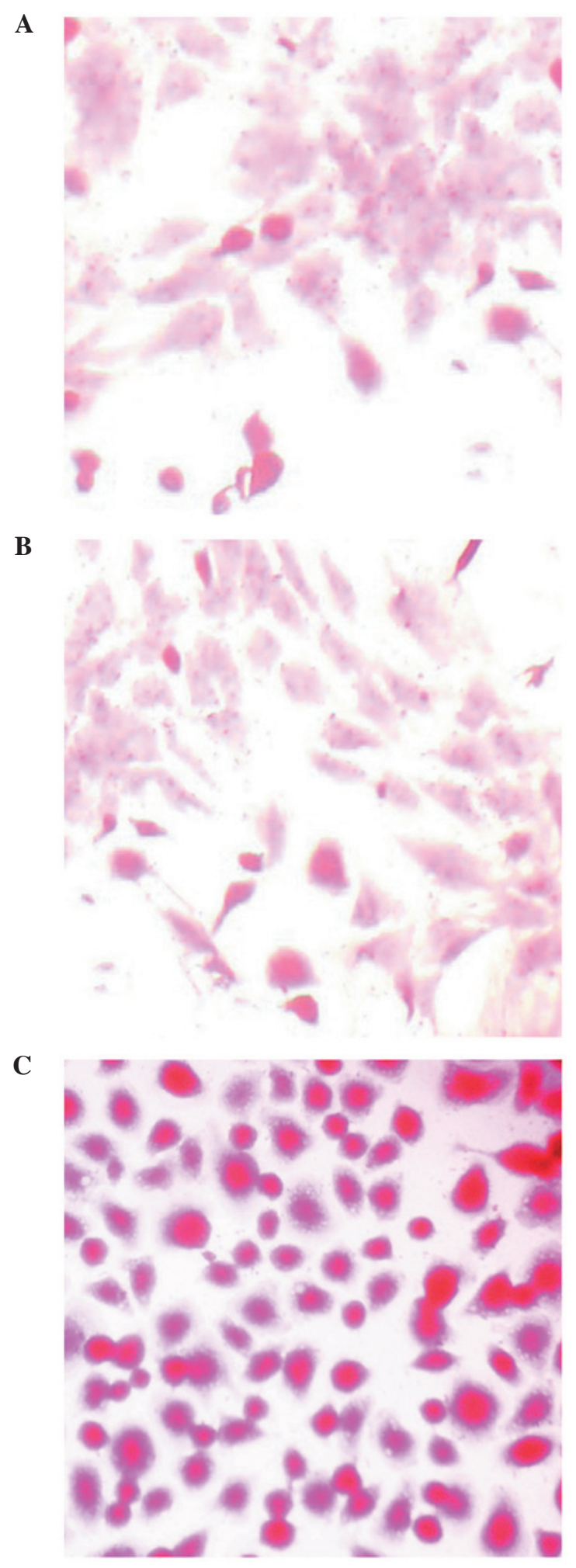

Figure 1. Liver cells for regular PAS staining. (A) Pancreatic enzyme digestion method; (B) liver cell grinding method; (C) organization block adherent method. The best serum concentrations of cultivated cells following PAS (magnification, x100). PAS, periodic acid-Schiff.

band of $\sim 400 \mathrm{bp}$. The brightness of the band from the normal cells, para-carcinoma cells and cancer cells markedly increased.

The Image $\mathbf{J}$ software was used to analyze each sample and the corresponding reference grey value respectively, and to calculate the grey level ratio of each sample (Table IV). 

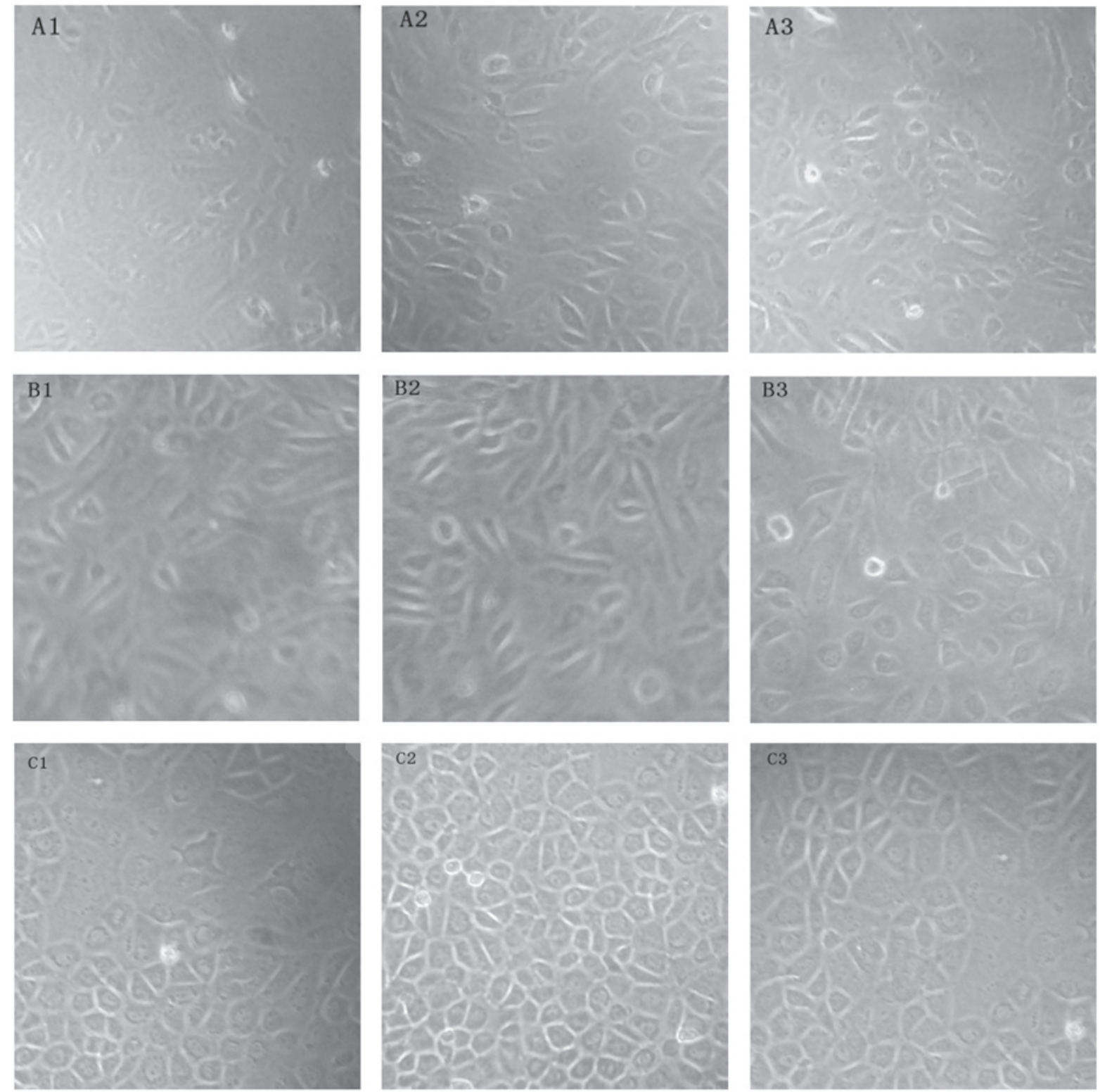

Figure 2. Observed groups of normal liver tissue cell culture cell morphology. (A1-A3) Pancreatic enzyme digestion method, serum concentrations of (A1) 10, (A2) 15 and (A3) $20 \%$ of cells (magnification, x100), respectively. (B1-B3) Liver cell grinding method, serum concentrations of (B1) 10, (B2) 15 and (B3) 20\% of cells (magnification, x100), respectively. (C1-C3) Organization block adherent method, serum concentrations of (C1) 10, (C2) 15 and (C3) $20 \%$ of cells (magnification, $\mathrm{x} 100$ ), respectively.

The electrophoresis results of the uPAR (D1D2) mRNA

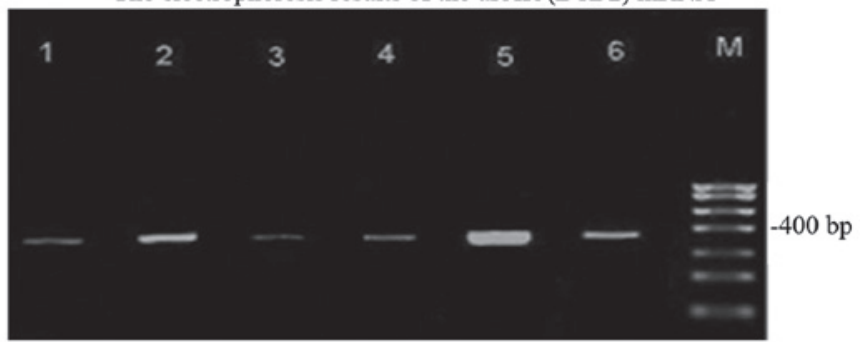

Figure 3. Lane $\mathrm{M}$ is the DNA marker, lanes 3 and 4 are normal liver cells, lanes 2 and 5 are liver cancer cells and lanes 1 and 6 are para-carcinoma cells.

Relationship between of the expression of $U P A R(D 1 D 2)$ in $H C C$ and the clinical pathological features. There was no association between the expression of UPAR (D1D2) in HCC, $\alpha$-fetoprotein (AFP), serum ferritin (SF), pathological grade and tumor size $(\mathrm{P}>0.05)$. However, it was associated with the DNA copy number $(\mathrm{P}<0.05$; Table $\mathrm{V})$

\section{Discussion}

Due to ethical issues, numerous human trials are not able to be performed. For this reason, as a research method in vitro, primary cell culture method has been widely used owing to its characteristic of maintaining human physiological functions in a certain period of time and obtaining stable metabolic product in a short period of time. Thus, obtaining cells with a high purity, high productivity and high activity is particularly important. At present, although there are several liver cell primary culture methods, certain experiments are not able to be performed in certain laboratories. 
In order to select a highly efficient, simple and economical liver cell primary culture method from normal liver tissue, cells were cultivated by the pancreatic enzyme digestion method, liver cell grinding method and organization block adherent method, respectively. The advantages and disadvantages of these three methods were evaluated by comparing the cell survival rate, purity and albumin supernatant fluid. Following evaluation, the tissue block adherent method with a $15 \%$ serum concentration was found to be the best liver cell culture method. This method is simple, efficient and economical. In addition, it obtains a high purity and high activity of liver parenchyma cells and provides sufficient stable samples for the follow-up study.

As an important component of the urokinase system, uPAR is able to combine with uPA on the surface of the cell, mediate signal transduction in cells and activate the intracellular protein kinase, and is important in the process of tumor growth and metastasis (7). mRNA splicing variants are one of the common features of malignant disease. However, it is not clear what causes tumor cell malignant transformation, by-products of cell transformation due to the tumor cells dysfunction or the increase of splicing variants themselves $(8,9)$. However, more and more evidence suggests that the mRNA associated with tumorigenesis may lead to cancer cells producing proteins with special functions, which is closely associated with the occurrence of cancer development. Splicing mutations may lead to the absence of certain regions of proteins, which in turn leads to a loss of function or a gain of function (10). A previous study demonstrated that more than half of human splicing variants of $\mathrm{G}$ protein coupled receptors code for functional proteins and the loss or increase of extracellular regions may be involved in protein interactions (11). A previous study reported that UPAR has numerous splice variants including uPAR del4/5, which lack exons 4 and 5 and mediates tumor-relevant biological processes in vivo and in vitro (12). In our previous experiments (13), following determining the serum content of uPA and UPAR in healthy individuals and liver cirrhosis and liver cancer patients by ELISA, we found that the expression levels of UPAR are closely associated with liver cancer development. UPAR splicing variant products were not determined in the experiments. The present study demonstrated that the expression of uPAR (D1D2) in hepatocellular carcinoma patients is significantly higher than in the control group, and is also higher in cancer tissue compared with para-carcinoma tissue. These results are consistent with the theory that the splicing variants of UPAR are increasingly expressed in other tumors. This suggests that D1D2 of UPAR is increasingly expressed during the development of liver cancer. There was no association between the expression D1D2 of UPAR in HCC and AFP, SF, pathological grading and tumor size $(\mathrm{P}>0.05)$, however, there was an association with the copy number of HBV DNA. The copy number of HBV DNA is used to distinguish the patients HBV replication level (14). In addition, chronic persistent infection of the virus increases the probability of integration of HBV DNA into liver cells. The continuous replication of HBV may activate certain proto-oncogenes and inactivate or mutate tumor-suppressor genes at the same time, promoting the occurrence of cancer. The integration of virus DNA is able to increase the HBV $\mathrm{X}$ antigen, inducing liver cell malignant transformation (15). By contrast, there are several splicing variants of UPAR and the conformational change of the UPAR isoform may lead to abnormal signaling transduction of uPAR (D1D2). The abnormal signal transduction of integrin $\alpha 5 \beta 1$ activates the activity of a series of downstream enzymes and activates the cell and the signaling molecules of the cell nucleus inside and outside, making liver cell differentiation to variation clonal hyperplasia, eventually leading to liver cancer. Mazzieri and Blasi (16) reported that the urokinase receptor is a multifunctional receptor, which regulates the dependence and independence process of proteins. Urokinase receptors, including integrin and epidermal growth factor receptor combines with extracellular protease urokinase, joining the lateral interactions of transmembrane receptors. Start the cascade effect of protein at the same time on the extracellular matrix components, the activated receptors of adjusting the important signal transduction are not only involved in the interaction of the modulation of the extracellular matrix, but also control extracellular signaling in order to determine cell proliferation.

In conclusion, although several studies regarding the association between varous splicing variants of UPAR and several types of diseases $(17,18)$ have been reported, studies concerning the association of uPAR (D1D2) with liver cancer have not yet been reported in the literature. The present study demonstrated that the expression of the urokinase receptor isoform exons UPAR (D1D2) increases from normal liver cells, liver cells to para-carcinoma cells. Therefore, the present study inferred that the splicing variation of UPAR (D1D2) is possibly associated with the incidence and development of liver cancer.

\section{Acknowledgements}

We would like to to express our heartfelt thanks to Professor Yuanjiao Huang of the Experiment Center of Guangxi Medical University for assistance with our study.

\section{References}

1. Mondino A and Blasi F: uPA and UPAR in fibrinolysis, immunity and pathology. Trends Immunol 25: 450-455, 2004.

2. Blasi F and Carmeliet P: uPAR: a versatile signalling orchestrator. Nat Rev Mol Cell Biol 3: 932-943, 2002.

3. Piironen T, Laursen B, Pass J, et al: Specific immunoassays for detection of intact and cleaved forms of the urokinase receptor. Clin Chem 50: 2059-2068, 2004

4. Behrendt N, Ploug M, Patthy L, et al: The ligand-binding domain of the cell surface receptor for urokinase-type plasminogen activator. J Biol Chem 266: 7842-7847, 1991.

5. Behrendt N, Ronne E and Dano K: Domain interplay in the urokinase receptor. Requirement for the third domain in high affinity ligand binding and demonstration of ligand contact sites in distinct receptor domains. J Biol Chem 271: 22885-22894, 1996.

6. Stewart CE and Sayers I: Characterisation of urokinase plasminogen activator receptor variants in human airway and peripheral cells. BMC Mol Biol 10: 75, 2009.

7. Andreasen PA, Kjøller L, Christensen L, et al: The urokinase-type plasminogen activator system in cancer metastasis: a review. Int J Cancer 72: 1-22, 1997.

8. Guo Z, Yang X, Sun F, et al: A novel androgen receptor splice variant is up-regulated during prostate cancer progression and promotes androgen depletion-resistant growth. Cancer Res 69: 2305-2313, 2009.

9. Fackenthal JD and Godley LA: Aberrant RNA splicing and its functional consequences in cancer cells. Dis Model Mech 1: 37-42, 2008. 
10. Keren H, Lev-Maor G and Ast G: Alternative splicing and evolution: diversification, exon definition and function. Nat Rev Genet 11: 345-355, 2010.

11. Bjarnadóttir TK, Geirardsdóttir K, Ingemansson M, et al: Identification of novel splice variants of Adhesion G protein-coupled receptors. Gene 387: 38-48, 2007.

12. Sato S, Kopitz C, Grismayer B, et al: Overexpression of the urokinase receptor mRNA splice variant uPAR-del4/5 affects tumor-associated processes of breast cancer cells in vitro and in vivo. Breast Cancer Res Treat 127: 649-657, 2011.

13. Zhan L, Lv X, Li S, Jiang H, Zuo J and Tang J: The correlation of liver cirrhosis plasma, uPA, uPAR level between liver fibrosis and carcinogenesis. J Clin Lab Sci 26: 144-145, 2008 (In Chinese).

14. Chu CJ, Hussain M and Lok AS: Quantitative serum HBV DNA levels during different stages of chronic hepatitis B infection. Hepatology 36: 1408-1415, 2002.
15. Su Q, Schröder CH, Hofmann WJ, et al: Expression of hepatitis B virus X protein in HBV-infected human livers and hepatocellular carcinomas. Hepatology 27: 1109-1120, 1998

16. Mazzieri R and Blasi F: The urokinase receptor and the regulation of cell proliferation. Thromb Haemost 93: 641-646, 2005.

17. Luther T, Kotzsch M, Meye A, et al: Identification of a novel urokinase receptor splice variant and its prognostic relevance in breast cancer. Thromb Haemost 89: 705-717, 2003.

18. Thurison T, Lomholt AF, Rasch MG, et al: A new assay for measurement of the liberated domain I of the urokinase receptor in plasma improves the prediction of survival in colorectal cancer. Clin Chem 56: 1636-1640, 2010. 